\title{
The Effects of Health Educational Lessons using Learning Activities that Make Students Apply The Knowledge
}

\author{
Hideaki Tanimoto \\ Faculty of Sport and Health Sciences \\ Osaka SangyoUniversity \\ Osaka, Japan \\ tanimoto-h@spo.osaka sandai.ac.jp
}

\begin{abstract}
The Japanese Education Ministry guidelines were revised in 2008 with an emphasis to elevate the thinking levels, judgment capacity and an expressive faculty. This will be passed down by the next guidelines revised in 2017. As in any health education, handling the Content of the Education Ministry guidelines on Health education and teaching methods should be devised by incorporating learning activities that make students apply acquired knowledge. As an example of the previous guideline description, the Education Ministry introduced learning activities, which taps into knowledge, creates thinking ability, a capacity for judgment and an expressive faculty. The purpose of this study is to examine the effects of health educational lessons using learning activities that make students apply knowledge. The study subjects were comprised of 78 Grades 3junior high school students, which took a lesson about drug abuse prevention using role-playing as a learning activity that makes the students use the knowledge. The results indicated that students notice the importance and difficulty applying the knowledge they learnt.
\end{abstract}

Keywords_ prevention of drug abuse, role-playing

\section{INTRODUCTION}

Since the 2008 revisions, Japan's Educational Guidelines have strongly emphasized on the development of "thinking power, judgment power, and expression power." Similarly, in health education, in "addressing content in the health field," the Guidelines state, "When teaching in the health field, we will create teaching methods such as incorporating learning activities and utilizing knowledge." As an explanation, it states, "this shows that by emphasizing the acquisition of knowledge, we cultivate thinking power, judgment power, and other skills by actively conducting learning activities and utilizing knowledge. When teaching, we will use practice, experiments, and task-based learning such as discussion using example cases, brainstorming, and cardiopulmonary resuscitation." That is, the Japanese health education recommends introducing new learning activities in health classes that utilize knowledge as a method for cultivating "thinking power, judgment power, and expression power." Therefore, in this research, we incorporated learning activities utilizing knowledge in "drug abuse prevention and health" in health education classes and verified the outcomes.

Drug abuse in Japan decreased in age over the years among high school and middle school students. In 2015,
Cases of sixth graders smoking cannabis were reported (Asahi Shimbun, November 10, 2015). Thus, drug abuse is not something that happens to everyone; rather occurs in the daily lives of children and students. Moreover, regarding use of stimulants through conventional intravenous injection, there is an attempt to shift from a dark and shady image to a lighter one by using fashionable monikers for stimulants such as "speed," "S," "ice," or "slimming drugs," or with alternative methods such as vaping or taking pills such as MDMA. These methods and names weaken resistance to drugs, fear, and guilt amongst children and students with knowledge about the fears of drug abuse from schools' from drug abuse prevention classes. However, the shift to lighter-sounding names and methods does not change the physical and psychological damages or the pharmacological actions from the abuse of drugs it remains the same.

In addition to the decrease in the age of drug users, the drug abuse situation in Japan has become increasingly sophisticated. It is urgent we ensure children's safety and health by generating strategies to help them acquire knowledge through education to protect them from these drugs.

In this research, in addition to learning about the dangers of drug abuse in the health education topic "drug" abuse prevention and health," we incorporated some activities to help them reject drugs and countermeasures for times when drug abuse is suggested to them through roleplaying. Next, we made efforts to verify the impact of these activities on the students.

Additionally, keeping in mind that all items above are for students interested in becoming health and physical education teachers, we also tried to cultivate human resources who could be responsible for Japan's health and safety education in the future by having them create a class plan.

\section{METHOD}

\section{A. Subjects}

The subjects were 78 students in 3 classes of ninth graders attending a public middle school (male: 41 students, female: 37 students). The middle school is located in the suburbs of Osaka prefecture. It is a small school with a total 
student population of 238, 38 faculty and staff members, and 2-3 classes per grade. There are 2 health education faculty members ( 1 male and 1 female)

\section{B. Class content}

We used role playing as a learning activity utilizing knowledge in classes on "drug abuse prevention and health." In this unit, tobacco, alcohol, and drug abuse and health were included in study of healthy lifestyles and disease prevention. This unit is said to be implemented with a goal of following the Middle School Educational Guidelines of Studying Health and Physical Education Edition (2008):

The use of stimulants and cannabis induces states of severe and sudden confusion accompanied by hallucination and possibly, sudden death. Dependency symptoms appear as a result of continuous drug use. This explains why when the drug use is discontinued or interrupted, various disturbances such as mental and physical suffering occurs.

Drug abuse not only interferes with healthy physical development and character building, it also hinders the ability to adapt to the society and develop a sense of responsibility. Therefore, it is logical that it leads to serious impacts on households, schools, and neighborhood communities as well as problems like violence, sexual misbehavior, and crime.

It is clear that behaviors such as drug abuse is promoted by psychological states such as curiosity, careless/hopeless attitudes, excessive stress, influence of surrounding people, and the social environment such as difficulty saying no in interpersonal relationships, advertisements, and ease of availability. Henceforth, we can also understand the necessity of appropriate countermeasures. [1]

Based on the information above, we prepared the following lesson plan (Table 1). Additionally, among the above-mentioned goals regarding drug abuse, it is promoted by psychological states such as curiosity, careless/hopeless attitudes, excessive stress, the influence of surrounding people, and the social environment such as difficulty saying no in interpersonal relationships, advertisements, and ease of availability, since cases of acquiring drugs from friends or acquaintances accounts for $45 \%$ of all drug acquisition (National Institute of Mental Health, 2016), we were able to teach students how to reject drugs when offered by a friend or acquaintance through role playing.

The atmosphere was different from usual with these targeted middle schoolers, some students felt confused at first or nervous. However, the students' impressions revealed that they were able to learn under this good tension.

\section{Measurement}

To confirm the outcomes of these classroom practices, we conducted a free-description questionnaire. It was titled "Impressions of This Course" and students described their impressions of the class and what they learned.

\section{Class CONDITIONS}

In this lesson, students who wish to teach plans and implement classes become the main subject. Since the our usual teacher is Mr. Yomura, but teachers came from Osaka
Sangyo University and it felt fresh and new. It felt different from usual and I thought it was great.

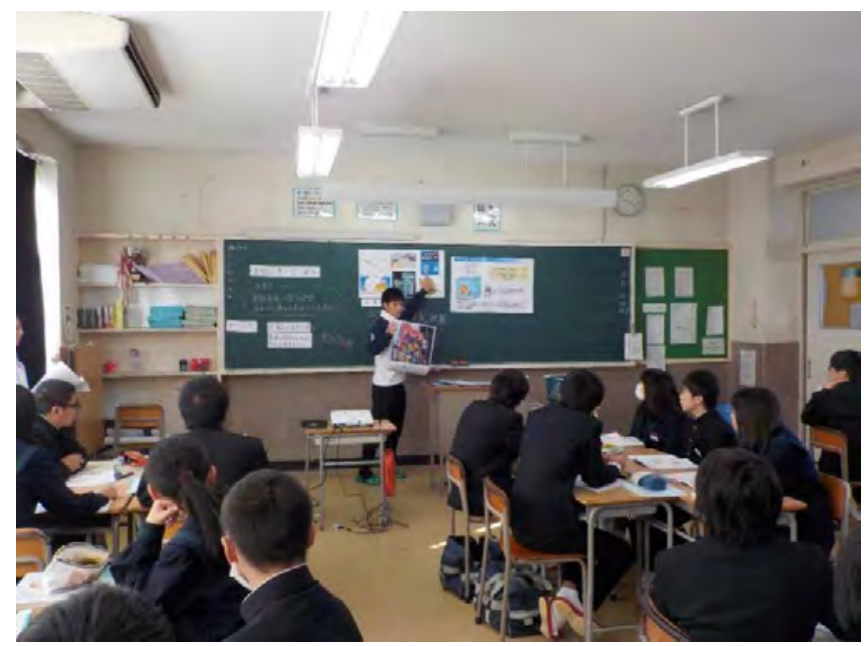

Fig. 1. A class in session

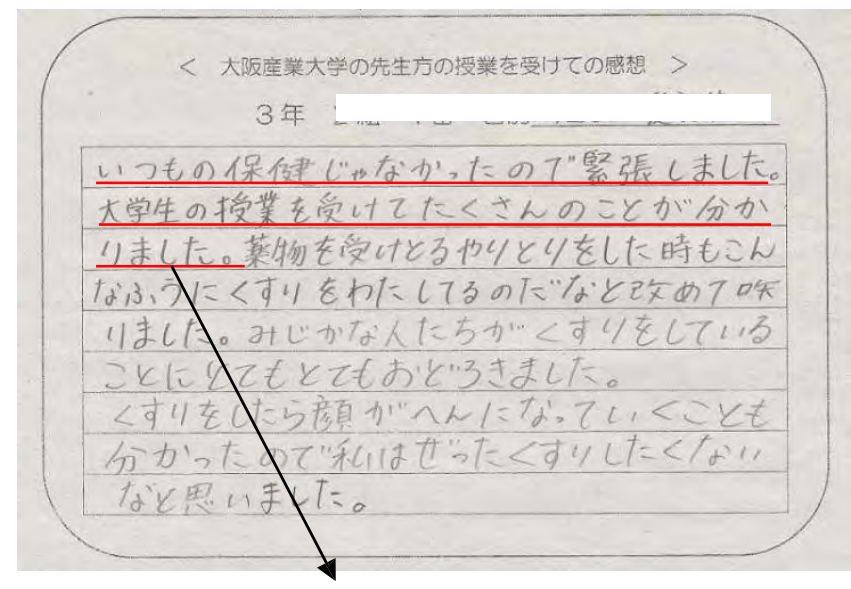

I was nervous because it was not the usual health class. I took the university students' class and I learned a lot.

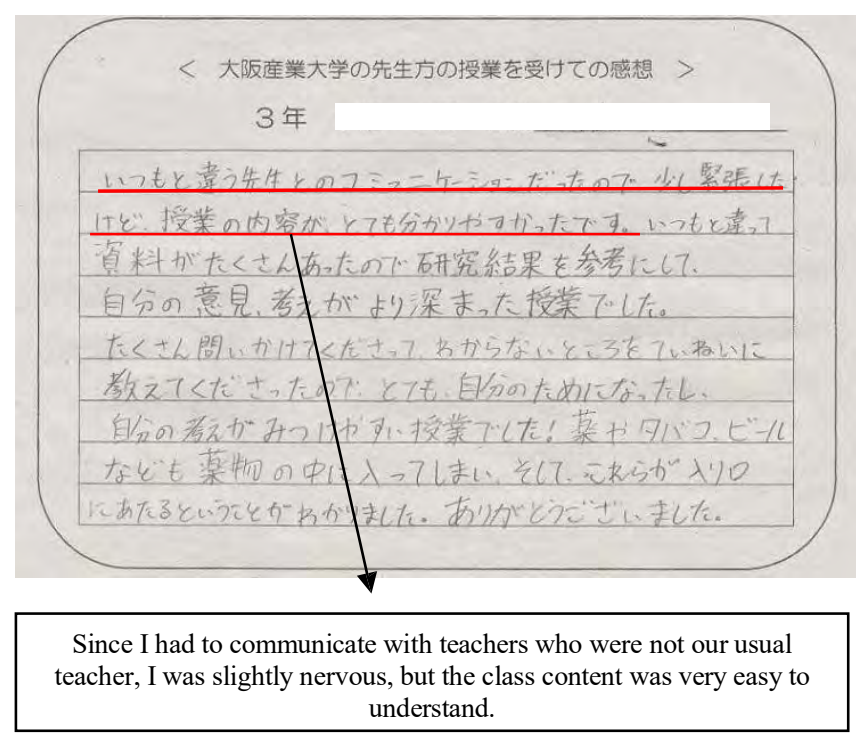




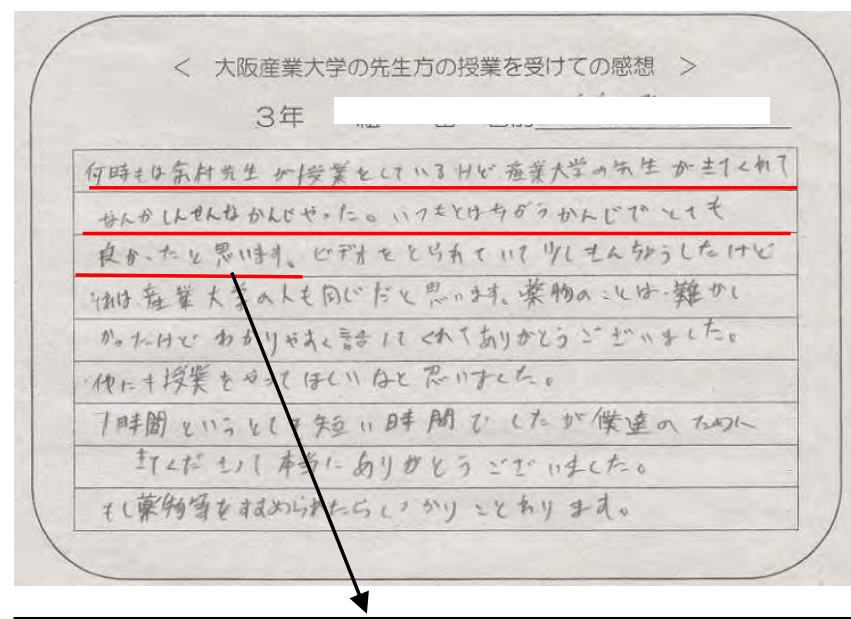

Our usual teacher is Mr. Yomura, but teachers came from Osaka Sangyo University and it felt fresh and new.

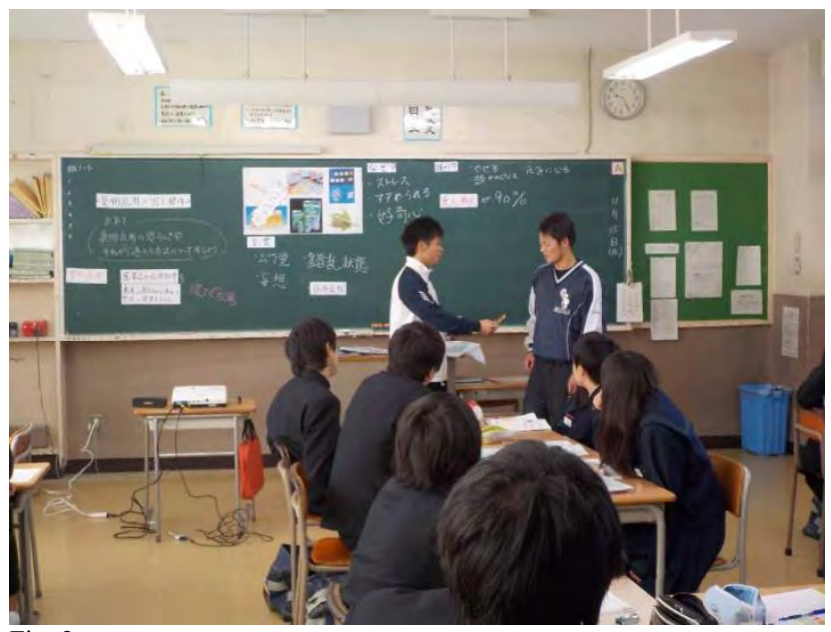

Fig. 2. Demonstration about role playing

Additionally, in the role-playing, which was the core of this practice, university students conducted a demonstration to enable students understand the methods of implementation. After the demonstration, students got in pairs or groups and separated into roles "friend offering drugs" and "refusing drugs." Then they practiced refusing drugs. To keep these activities moving, the university students observed the pair learning and participated in roleplaying with the students as needed.

\section{Class ACHIEVEMENT}

After this class, students were given the "impressions after taking this class" questionnaire and they freely described their impressions of the class and what they learned. After confirming content of the questionnaires, we found that many students described "fear of drugs affecting their mind and body" and "refusing drugs when offered."

The following descriptions were given regarding "fear of drugs affecting their mind and body."

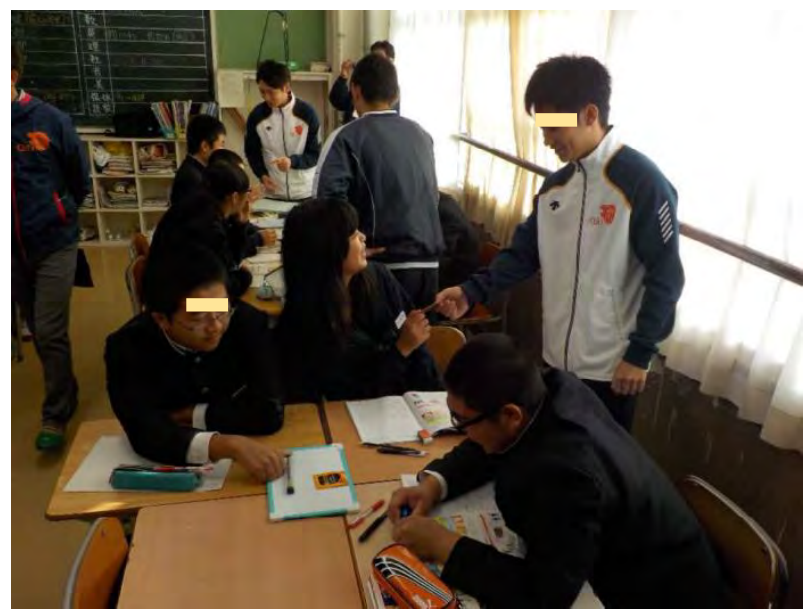

Fig. 3. Students try role playing

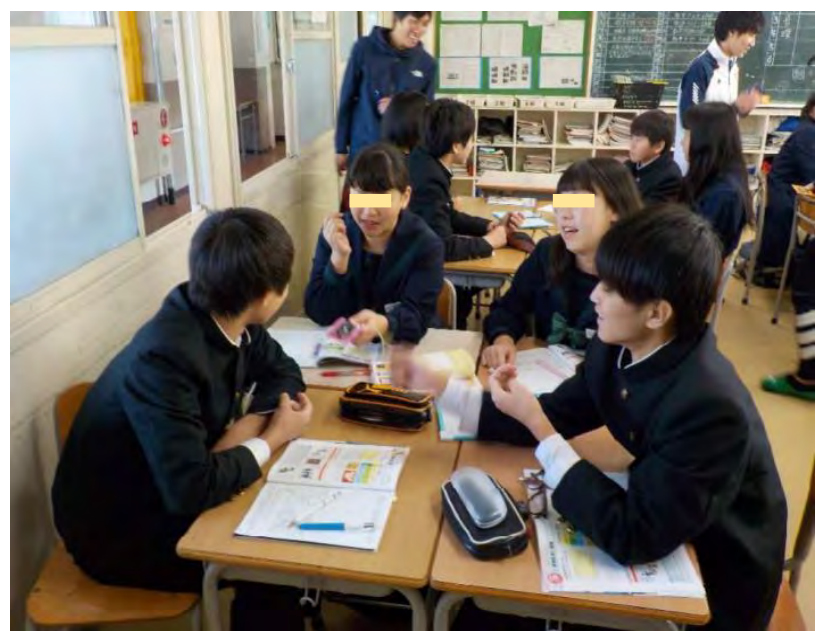

Fig. 4. Role playing with university student

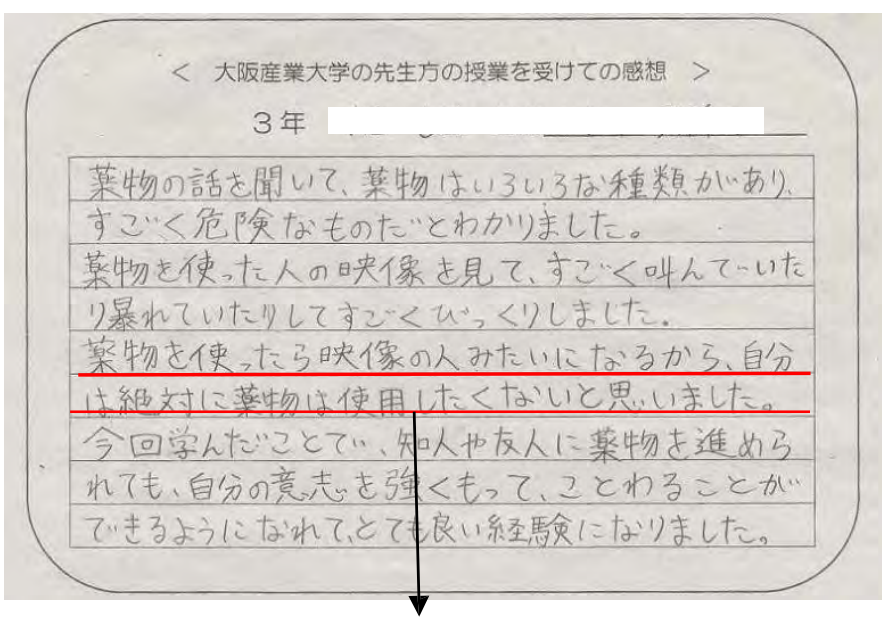

If I used drugs, I would become like the person in the movie, and so I absolutely do not want to use drugs. 


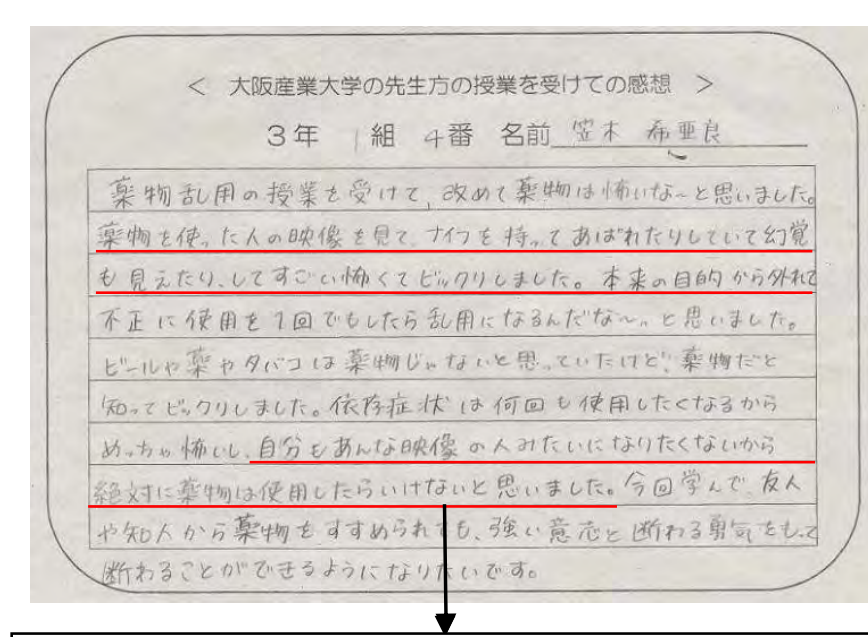

I thought drugs were scary. Watching the movie of the person using drugs, I was really scared and shocked that the person went on a rampage with a knife and was hallucinating. I do not want to become like the person in the movie, so I absolutely cannot use drugs.

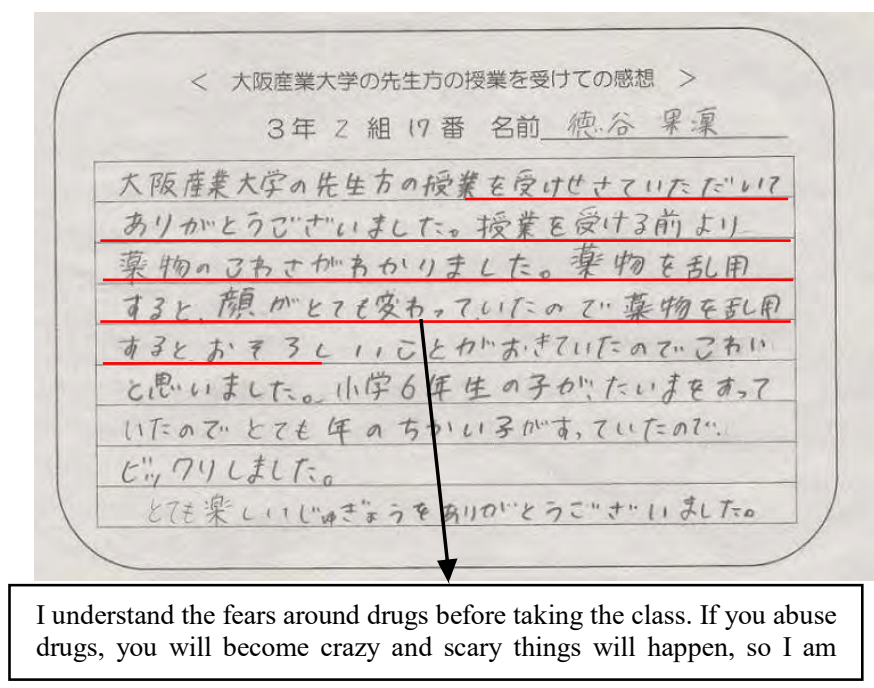

In this project, based on the images created by Fukuoka prefecture to the raise awareness of drug abuse prevention, we created a visual understanding of the kinds of states drug abusers fall into. It is assumed that as a result, students who took the class experienced the fear of drug abuse.

The following descriptions were given regarding "refusing drugs when offered."

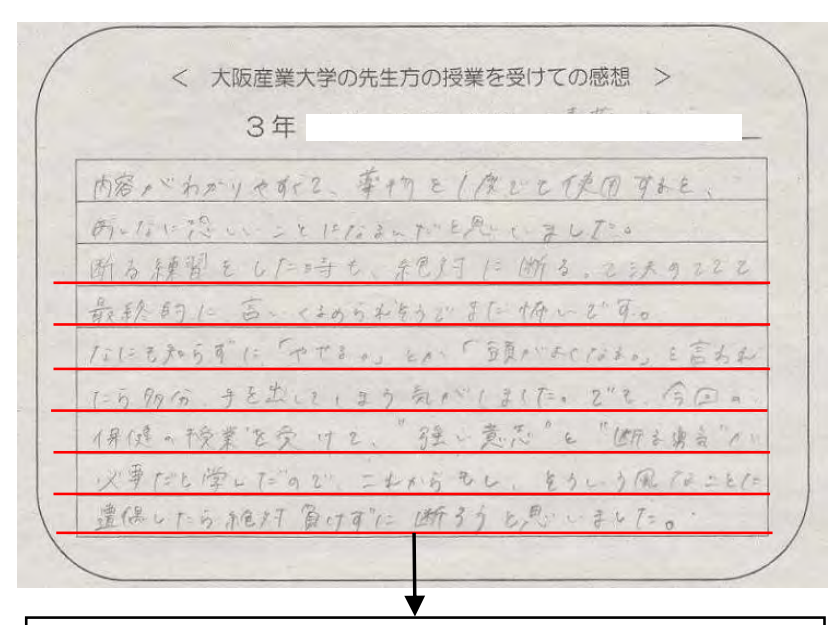

Even when practicing refusing, when I was absolute sure I would refuse, at the end it seemed I might be talked into it, so I was still scared. I felt like if I did not know anything and I was told, "You'll lose weight" or "you'll become smarter," I would probably accept the drugs. However, after taking this health class, I learned how important it is to have a "strong will" and "courage to refuse," so in the future, if I encounter this kind of thing, I think I will absolutely refuse.

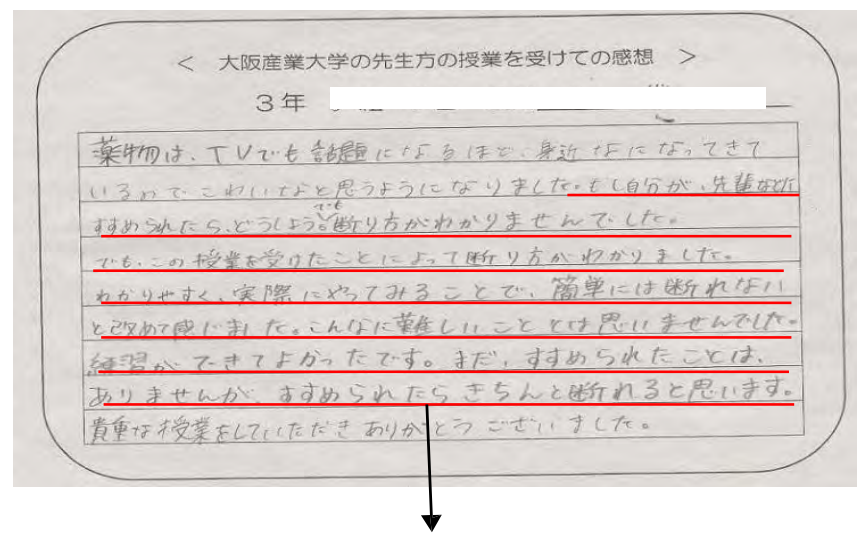

What if senior classmates offered me drugs? I did not know how to refuse. Now I understand how to refuse after taking this class. By actually practicing, I felt again that I could not easily refuse. I did not think it was that hard. I am glad I got to practice. I have not been offered yet, but if I were, I would be able to rightly refuse.

Based on the descriptions above, students who took this class fully understood the effects of drug abuse on the mind and body and it seems that they formed a strong against drug abuse. Additionally, although some students properly learnt how to refuse when invited to abuse drugs, there were also many students who were unable to learn how to refuse. Yet, these students realized the difficulty of refusing and it seems that they felt the importance of learning these ways to refuse. That is, most students did not just acquire knowledge about substance abuse, it can be said that they were also able to learn how to utilize acquired knowledge.

In this research, however, since we are studying the outcomes of this class only through data received from the free-description responses on post-class impressions, it is unlikely that generalizations can be made. In the future, to enhance the versatility of these classroom practices, it is 
critical to conduct a longitudinal study while gathering objective data.

\section{REFERENCES}

[1] Ministry of Education, Culture, Sports, Science and Technology. "Exposition of ministry's curriculum guideline about physical education", 2008.
[2] Ministry of Education, Culture, Sports, Science and Technology. "Exposition of ministry's curriculum guideline about physical education", 2017.

[3] T. Shimane, M. Omagari, K. Kitagaki, H. Tachimori, D. Chu, K. Wada, 'National research of junior high school students' consciousness and actual conditions about alcohol drinking, smoking and drug abuse". Research report of Health and Labour Sciences Research Grant, 2016. 